\title{
Multi Camera for Surveillance System Ground Detection and 3D Reconstruction
}

\author{
Xu Yongzhe ${ }^{1}$ and Byungsoo Lee ${ }^{1}$ \\ 1Department of Computer Engineering, University of Incheon, Korea \\ yongzhexu@hotmail.com,bsl@incheon.ac.kr
}

\begin{abstract}
In this paper, a method for detection ground coordinate to surveillance camera system in a real-time. video capture information from surveillance camera system with SFM(stricter from motion) algorithm presented. $3 D$ reconstruction is rebuilding $3 D$ geometric from multi $2 d$ images. Researcher focus on 3D recognize and matching algorithm. $1^{\text {st }}$ surveillance camera system detection ground mark image. $2^{\text {nd }}$ matching image pattern and compute mark image coordinate information, $3^{\text {rd }}$ compute information combine with position information, $4^{\text {th }}$ reconstruction in $3 D$ space surveillance environment.
\end{abstract}

Keywords: surveillance camera system, reconstruction environment, SFM, image matching

\section{Introduction}

The problem detection surveillance system ground is receiving increased object pose in many including surveillance area. Robust detection of ground in receiving video is important for many surveillance camera system, such as surveillance area, smart rooms, structure from motion room, intelligent living environment, and human trajectory analysis[1]. In this paper we address the case of multi camera detection ground plane and compute these position information to $3 \mathrm{D}$ environment. The computed result help for improvement surveillance camera system illumination noise, object position, object detection. The goal of the research presented in this paper is to automatically detect and track a coordinate information of target object in complex experiment environment. On the other hand, surveillance camera system need provide coordinate compute information from the complex environment. Proposed algorithm have two limitations, however complex environment create pattern miss matching, illumination changing need more experiment result, compute all input video need more high speed operation ability[1].

\section{Related Work}

During the last decade, surveillance camera systems have established to become the standard and one of popular efficient sensorial assets in industrial and every Security Stakeholders. Other researcher focus on detection ground plane have many algorithm, such as visual navigation, computer vision based driving assistance, and 3D environment map construction. From paper "Detailed 3D Representations for Object Recognition and Modeling" main target is like image 1 find point cloud data set. After find object world coordinate detection ground and tracking object from single camera $[2,3,4]$. Figure 1 shows object recognition and modeling find ground detection algorithm. 


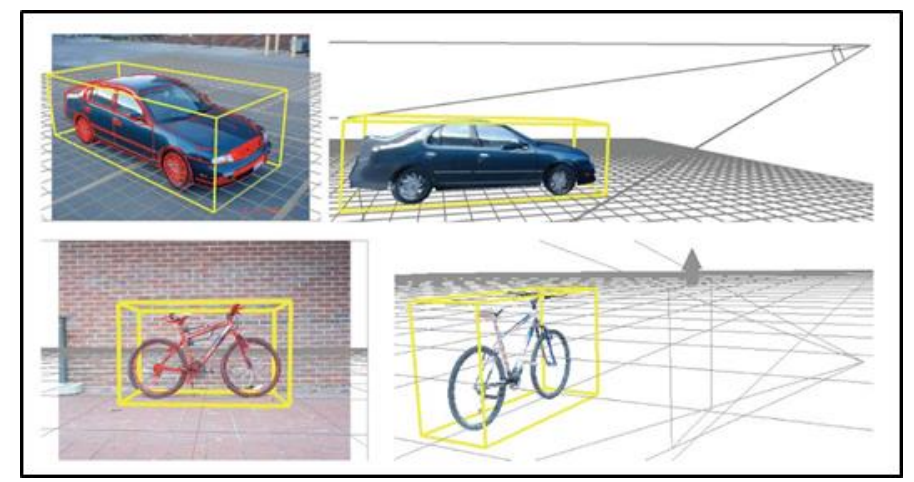

Figure 1. Cloud Point Feature Detection from 2D Image

\subsection{Hough Line Detection Algorithm in Detection Ground}

Figure 2 shows Hough line detection is Opencv basic algorithm. It is detection straight lines and apply the transform, first an edge detection pre-processing is desirable. A line in image space can be expressed with two variables. 1st transform color image to gray image. 2nd find image edge feature point. But this algorithm have illumination problem like figure 3. Illumination change ground line edge. It is impossible to find detection line in environment.

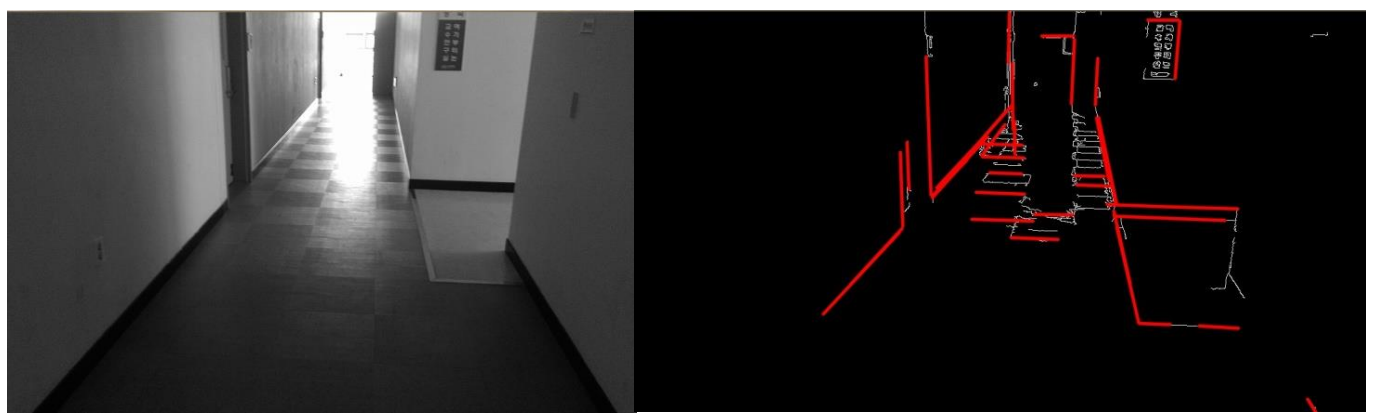

Figure 2. Hough Line Detection Object

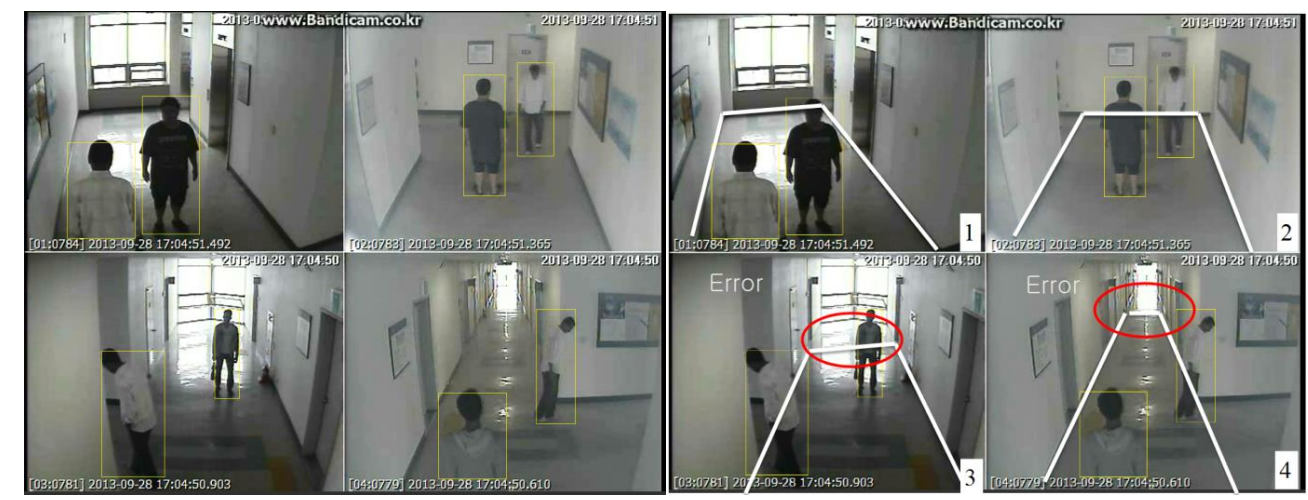

Figure 3. Hough Line Detection Problem

\subsection{Point Cloud Algorithm}

Point cloud Algorithm (other researcher achievement) Point cloud algorithm is find same feature point transform coordinate to 3D world space. Paper "A Fast kNeighborhood Algorithm for Large Point-Clouds" improves these coordinate more realities[5, 6, 7]. Figure 3 shows Point cloud algorithm to reconstruction 3D object. The 
point cloud library is a standalone, large scale, open project 2D/3D image and point cloud processing. It is released under the terms of the BSD license, free for research use. It supported by a consortium of commercial companies, organization, open perception. In this paper we used 2D part and transform 2D coordinate to 3D coordinate library from point cloud library.

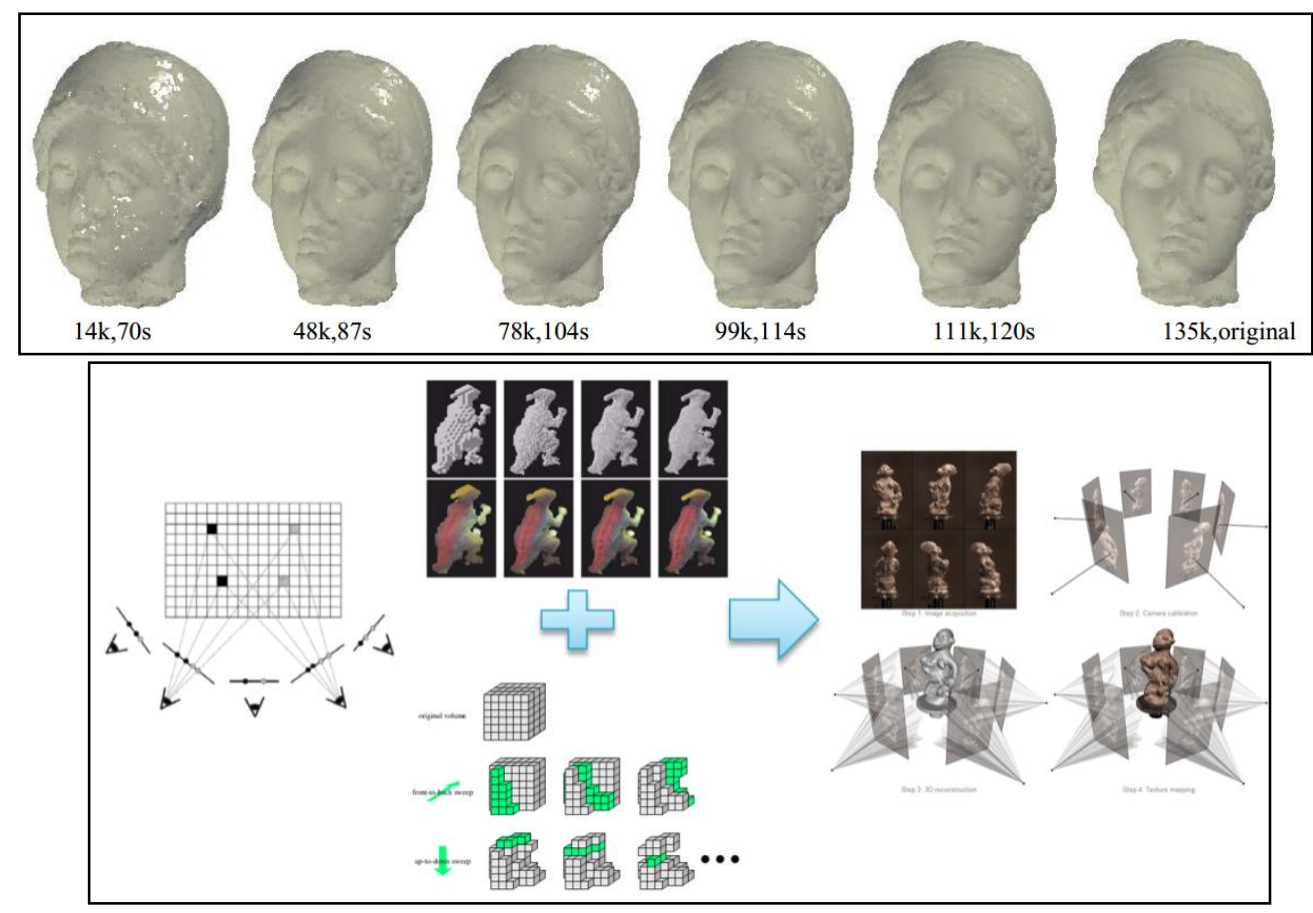

Figure 4. Object Structure from Multi Images(b)

3D Reconstruction SFM (structure from motion) is rebuilding 3D geometric from multi 2D images. Researcher focuses on 3D recognize and matching algorithm. Researching algorithm main target is from 2D image object silhouette find object pose (Target Object $\mathrm{X}, \mathrm{Y}, \mathrm{Z}$ world coordinate). Then compute object pose easy to compute ground. After detection ground finds wall, building and other static object. Transform these objects coordinate to $3 \mathrm{D}$ environment to 3D space coordinate.

This research Main goal is how to reconstruction world ground from multi surveillance 2D cameras. The experiment approach is multi surveillance camera system capture target object on ground. These images information had object silhouette and real world coordinate and position. Research final goal is analysis this information and compute it to $3 \mathrm{D}$ environment

\subsection{Main Algorithm Feature Point Matching}

Image 5 show main algorithm feature point matching the step 1st is from input device capture target object. 2nd find features from object. 3rd matching all these feature point. The last step structure from motion find same feature point and mark it and transform plane coordinate to game engine world coordinate. 


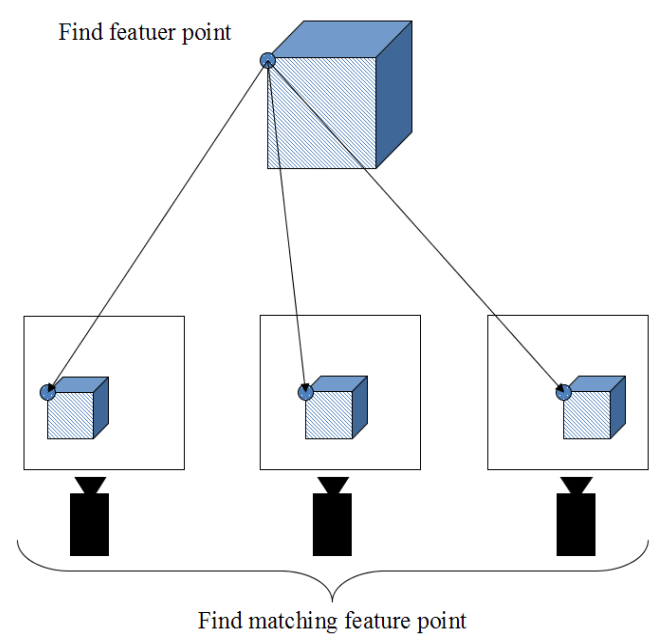

Figure 5. Multi Camera Edge Feature Point Matching
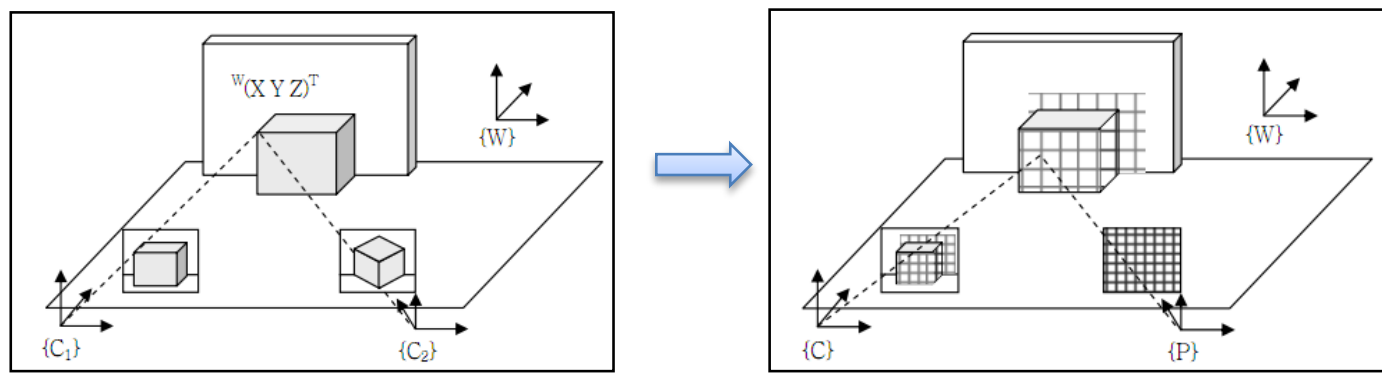

Figure 6. Target Object Block Divide

Figure 6 shows After previous step camera 1, 2 focusing object of world transform(X, $\mathrm{Y}$, and Z) left camera and right camera detection object divide object to small block. Object block silhouette matching to 3D environment. Figure 3 shows object block structure these points coordinate in $3 \mathrm{D}$ environment $[8,9]$.

\subsection{Previous Proposed Algorithm Design}

Propose algorithm design is from 2D video capture images. Capture images using feature tracking and matching. After matching feature point transform to 3D Reconstruction to 3D game environment. Detection wall, ground, building and static object implement. Proposal algorithms are combining 2.1, 2.2, 2.3 algorithm to one camera surveillance system. That mean this improve algorithm is from capture image information to reconstruction 3D environment.

Surveillance camera system knows position coordinate information data it is easy to transform these position data to system. Figure 4 shows Roger Y.Tsai algorithm it 2 stage algorithm calculating the pose. 3D object and $\mathrm{x}$-axis and $\mathrm{y}$-axis translation is first stage in second stage it computes the focal length, distortion coefficients and the z-axis translation. Also it is calculating support Zhengyou Zhang " a flexible new technique for camera calibration", and this algorithm based on corner edge detection by chess board. Figure 7 shows if know about ground position information surveillance camera system easy to transform these position data to video plane pixel coordinate. Also these transform support multi camera system, find same feature matching algorithm[10, 11,12]. 


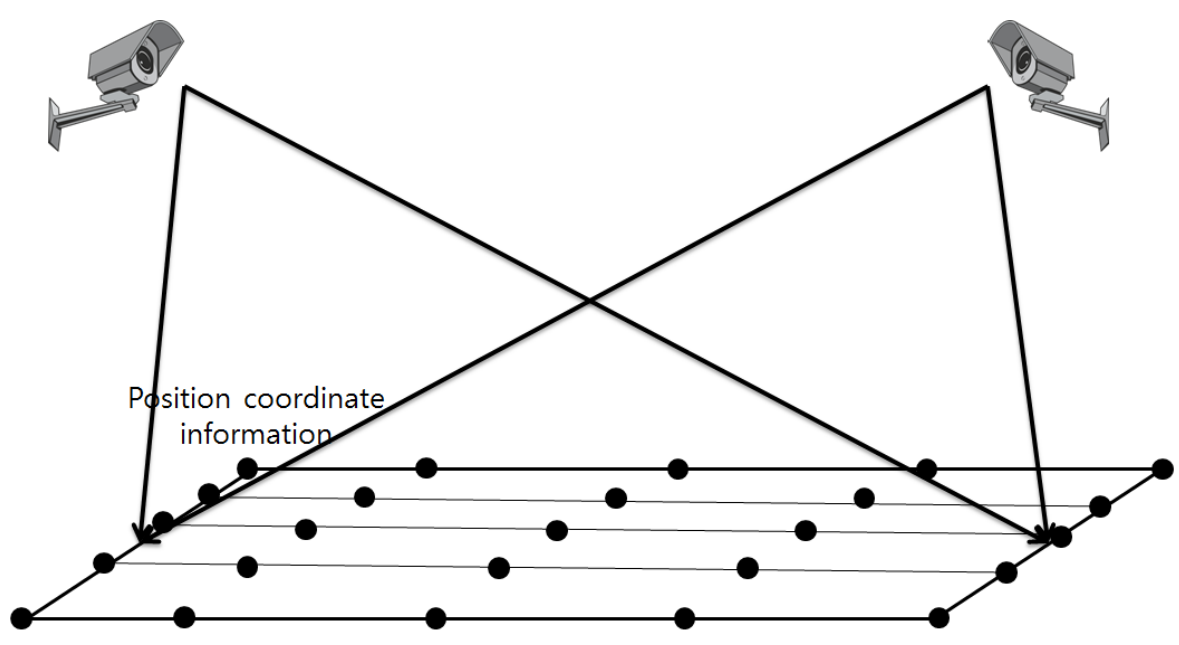

\section{Figure 7. Surveillance Camera System Position Coordinate Information Detection}

Figure 8 shows used pets 2007 coordinate information to calculate video plane in surveillance camera system. White line $-3,1$ left down coordinate information 3, 1 left top coordinate information 3, -2 right top coordinate information, $-3,-2$ right down coordinate information. All information from pets 2007 XML file calculate this data transform used point cloud library. All these XML parameter ex) focal lenz parameter, world coordinate, image plane coordinate, image pixel position transform position to each other parameter[13].

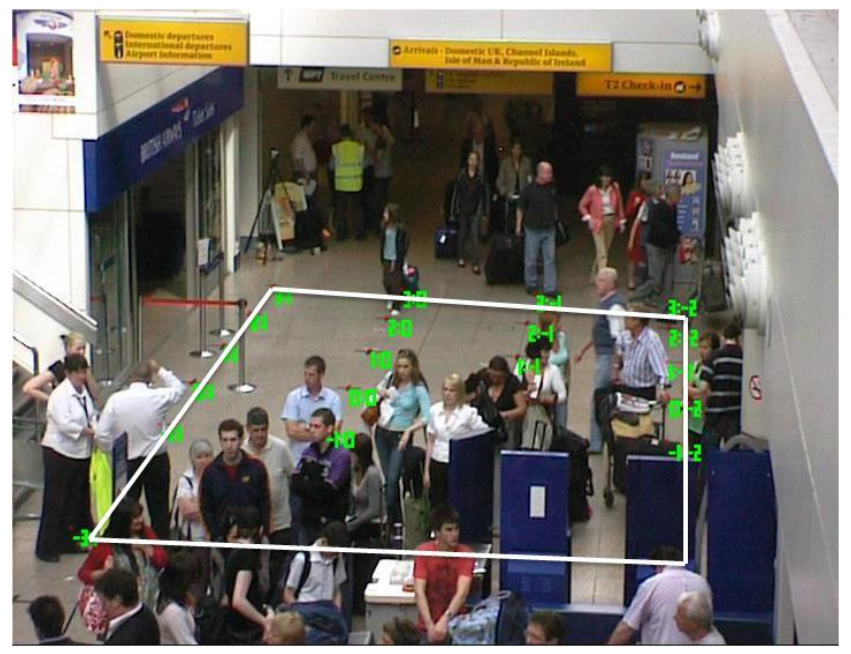

Figure 8. Pets 2007 Calculating Position Information

\subsection{Main Purpose of Algorithm}

This proposal algorithm from input surveillance camera system computing object world coordinate transform to $3 \mathrm{D}$ environment coordinates. Use coordinates information recognition object, reconstruction in 3D environment find ground, wall and building. These detection information improve surveillance system tracking moving object, illumination condition $[14,15]$. 


\section{Proposal Algorithm}

The research goal and necessity is to exploit the information of 3D Reconstruction SFM. SFM (structure from motion) is rebuilding 3D geometric from multi 2D images. We focuses on 3D recognize and matching algorithm. Algorithm main target is from 2D image object silhouette find object pose (Target Object X, Y, Z world coordinate). And compute object position to find about surveillance ground. After compute object pose easy to compute and reconstruction ground. Detection ground finds wall, building and other static object. Transform these objects coordinate to 3D environment ex) game engine, simulation tool, and CAD. .

\section{Experiment Result}

In the experiment result that we used color region algorithm to surveillance camera system area. Figure 7 left and right shows pets 2007 surveillance camera capture image to gray scale image. Pixel position transform computing did not need RGB color. 1st step change color video to gray scale video. Figure 8 left shows color region divide right shows $2 \mathrm{D}$ pixel position coordinate to video plane coordinate. And proposed algorithm change detection wall to yellow color. Ground detection algorithm based on pets 2007 XML detection ground plane [16, 17, $18]$.

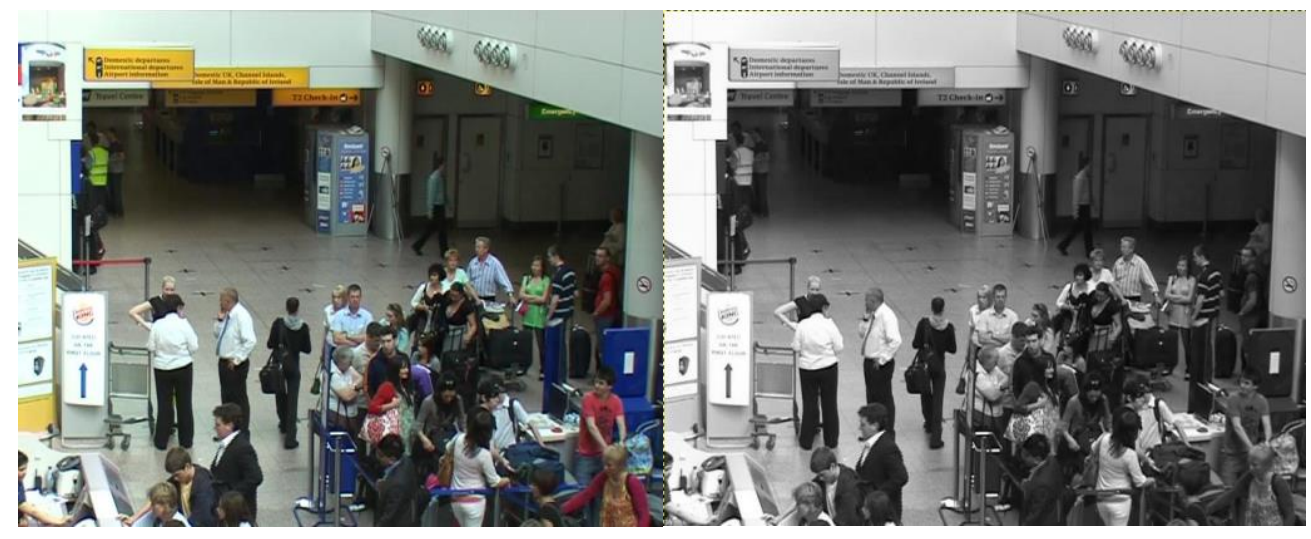

Figure 7. Pets 2007 Surveillance Camera Experiment Result Transform Color Video to Gray Video

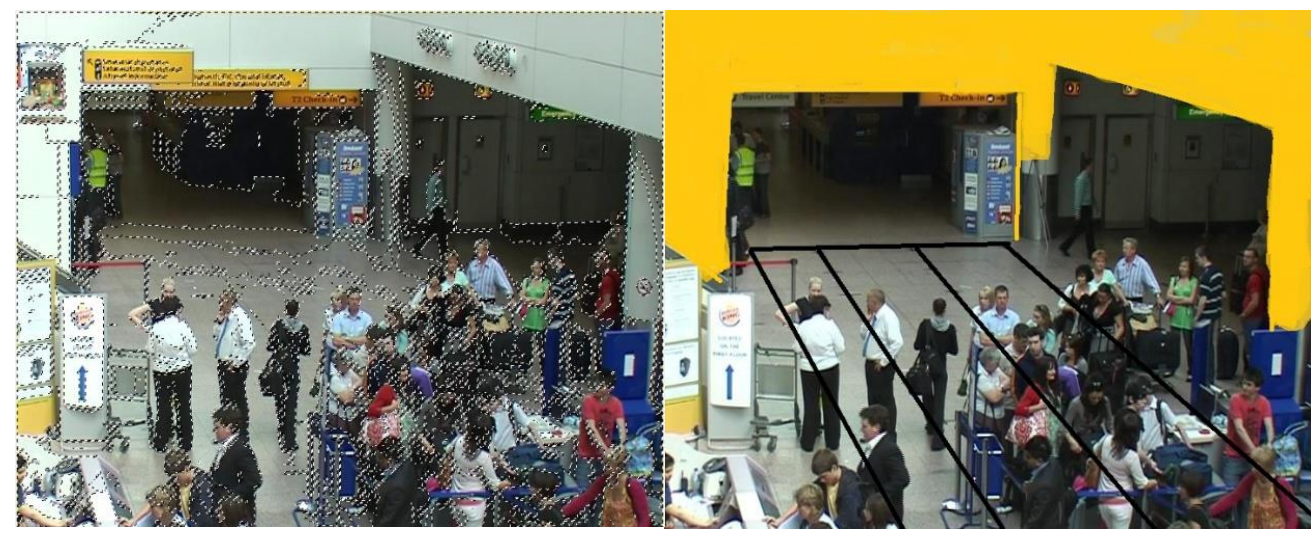

Figure 8. Pets 2007 (left) Color Region Divide (right) Transform Color Region to 3d World Coordinate 


\section{Conclusion and Future Work}

We have proposed algorithm based on background coordinate information and pets 2007 coordinate information from surveillance camera system. Algorithm is computing object world coordinate transform to 3D environment coordinates. Restrictive conditions of the Complex environment have some error. It is also have Illumination problem based on reconstruction 3D computing. Proposed algorithm challenging part in process, as computes the $3 \mathrm{D}$ object missing from feature matching. The challenging part is $3 \mathrm{D}$ word coordinate transform need pre-computing. Test video Pre-computing time average in $3 \sim 5$ hours.

\section{Acknowledgements}

This paper is a revised and expanded version of a paper entitled "Multi Camera Surveillance System for Ground Detection" presented at "XU YONGZHE", Hochiminh, Vietnam on October 24-26, 2014, NGCIT. This work was supported by Incheon National University Research Grant in 2014

\section{References}

[1] J. C. Kim, K. M. Lee, B. T. Choi and S. U. Lee, "A Dense Stereo Matching Using Two-Pass Dynamic Programming with Generalized Ground Control Points", Proceedings of the IEEE Computer Society Conference on Computer Vision and Pattern Recognition (CVPR'05), (2005).

[2] H. Grabner, J. Gall and L. Van Gool, "What Makes a Chair a Chair?", Proceedings of the IEEE Computer Society Conference on Computer Vision and Pattern Recognition (CVPR'11), (2011).

[3] K. Sakurada, T. Okatani and K. Deguchi, "Detecting Changes in 3D Structure of a Scene from Multiview Images Captured by a Vehicle-mounted Camera", Proceedings of the IEEE Computer Society Conference on Computer Vision and Pattern Recognition (CVPR'13), (2013)

[4] M. Z. Zia, M. Stark, B. Schiele and K. Schindler, "Detailed 3D Representations for Object Recognition and Modeling", IEEE Transactions on Pattern Analysis and Machine Intelligence, (2013).

[5] S. M. Seitz, B. Curless, J. Diebel, D. Scharstein and R. Szeliski "A Comparison and Evaluation of Multi-View Stereo Reconstruction Algorithms", Proceedings of the IEEE Computer Society Conference on Computer Vision and Pattern Recognition (CVPR'06), (2006).

[6] W. G. Choi, Y. W. Chao, C. Pantofaru and S. Savarese "Understanding Indoor Scenes using 3D Geometric Phrases", Proceedings of the IEEE Computer Society Conference on Computer Vision and Pattern Recognition (CVPR'13), (2013).

[7] M. Liu, R. Hartley and M. Salzmann, "Mirror Surface Reconstruction from a Single Image", Proceedings of the IEEE Computer Society Conference on Computer Vision and Pattern Recognition (CVPR'13), (2013).

[8] L. Del Pero, J. Bowdish, B. Kermgard, E. Hartley and K. Barnard "Understanding Bayesian rooms using composite 3D object models", Proceedings of the IEEE Computer Society Conference on Computer Vision and Pattern Recognition (CVPR'13), (2013).

[9] Z. Yang, Z. W. Xiong and Y. Y. Zhang, "Depth Acquisition from Density Modulated Binary Patterns", Proceedings of the IEEE Computer Society Conference on Computer Vision and Pattern Recognition (CVPR'13), (2013).

[10] H. Hirschmuller "Accurate and Efcient Stereo Processing by Semi-Global Matching and Mutual Information", IEEE Conference on Computer Vision and Pattern Recognition (CVPR), (2005);San Diego, CA, USA.

[11] T. Svoboda, H. Hug and L. J. V. Gool, "Viroom - low cost synchronized multicamera system and its self-calibration", in Proceedings of the $24^{\text {th }}$ DAGM Symposium on Pattern Recognition, (2002), pp. 515-522.

[12] D. Chrysostomou, N. Kyriakoulis and A. Gasteratos, "Multi-camera 3D Scene Reconstruction from Vanishing Points", Imaging Systems and Techniques (IST), IEEE International Conference on, (2010), pp. $343-348$.

[13] C. H. Lin, S. Y. Jiang, Y. J. Pu and K. T. Song, "Robust Ground Plane Detection for Obstacle Avoidance of Mobile Robots Using a Monocular Camera”, The IEEE/RSJ International Conference on Intelligent Robots and Systems, (2010).

[14] M. D. Breitenstein, F. Reichlin, B. Leibe, E. K. Meier, L. Van Gool, "Robust Tracking-by-Detection using a Detector Confidence Particle Filter", Computer Vision, IEEE 12th International Conference on, (2009).

[15] M. Andriluka, S. Roth and B. Schiele, "People tracking by detection and people detection by tracking", In CVPR, (2008). 
[16] M. D. Beynon, D. J. Van Hook, M. Seibert and A. Peacock, "Detecting Abandoned Packages in Multicamera Video Surveillance System", Advanced Video and Signal Based Surveillance, Proceedings, IEEE Conference, (2003).

[17] M. Harville, G. Gordon and J. Woodfill, "Foreground segmentation using adaptive mixture models in color and depth", In Proceedings of the IEEE Workshop on Detection and Recognition of Events in Video, (2001); Vancouver, Canada.

[18] T. Zhao, M. Aggarwal, R. Kumar and H. Sawhney, "Real-time Wide Area Multi-Camera Stereo Tracking”, CVPR, (2005).

\section{Authors}

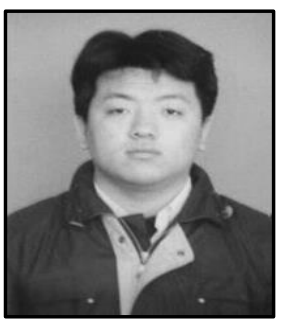

Yongzhe Xu received his MS in Computer science and engineering from Univesity of Incheon, Korea, in 2009.He is currently working toward a $\mathrm{PhD}$ in computer science and engineering at the same university. His research interests include computer graphic, FDS, virtualization, and Pattern recognition, Machine Learning.

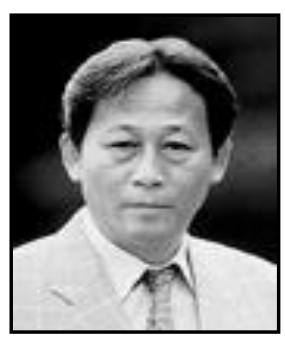

Byungsoo Lee received his MS in MBA from University of Dongguk, Korea ,1980. Received his Doctor of Science from University of KyongGi, Korea, $1998 \mathrm{He}$ is an Professor of Department of Computer Engineering at the University of Incheon, Korea. His research interests include software design, decision making system, eCRM, RFID/USN, IT convergence 